\title{
Exercise alters myostatin protein expression in sedentary and exercised streptozotocin-diabetic rats
}

Daniela Bassi', Patricia de Godoy Bueno², Keico Okino Nonaka², Heloisa Sobreiro Selistre-Araujo2, Angela Merice de Oliveira Leal ${ }^{3}$

${ }^{1}$ Departments of Physiotherapy, Federal University of São Carlos, São Carlos, SP, Brazil ${ }^{2}$ Departments of Physiology, UFSCar, São Carlos, SP, Brazil ${ }^{3}$ Departments of Medicine, UFSCar, São Carlos, SP, Brazil

\section{Correspondence to:} Angela Merice de Oliveira Leal Departamento de Medicina, Universidade Federal de São Carlos Rod. Washington Luís, km 235 13565-905 - São Carlos, SP, Brazil angelaleal@ufscar.br

Received on Sept/2/2014 Accepted on Feb/17/2015

\begin{abstract}
Objective: The aim of this study was to analyze the effect of exercise on the pattern of muscle myostatin (MSTN) protein expression in two important metabolic disorders, i.e., obesity and diabetes mellitus. Materials and methods: MSTN, is a negative regulator of skeletal muscle mass. We evaluated the effect of exercise on MSTN protein expression in diabetes mellitus and high fat diet-induced obesity. MSTN protein expression in gastrocnemius muscle was analyzed by Western Blot. $\mathrm{P}<0.05$ was assumed. Exercise induced a significant decrease in glycemia in both diabetic and obese animals. Results: The expression of precursor and processed protein forms of MSTN and the weight of gastrocnemius muscle did not vary in sedentary or exercised obese animals. Diabetes reduced gastrocnemius muscle weight in sedentary animals. However, gastrocnemius muscle weight increased in diabetic exercised animals. Both the precursor and processed forms of muscle MSTN protein were significantly higher in sedentary diabetic rats than in control rats. The precursor form was significantly lower in diabetic exercised animals than in diabetic sedentary animals. However, the processed form did not change. Conclusion: These results demonstrate that exercise can modulate the muscle expression of MSTN protein in diabetic rats and suggest that MSTN may be involved in energy homeostasis. Arch Endocrinol Metab. 2015;59(2):148-53
\end{abstract}

Keywords

Myostatin; exercise; diabetes; obesity; western blot

\section{INTRODUCTION}

$\mathrm{M}$ yostatin (MSTN) or growth and differentiation factor 8 (GDF8) is a member of the transforming growth factor- $\beta$ (TGF $\beta$ ) superfamily and has been characterized as a negative regulator of skeletal muscle mass in different (1). It is expressed mainly in muscle but also in adipose tissue (2). Following synthesis as a precursor protein, MSTN is processed by proteolysis by the furin family enzymes, with removal of the 24-amino acid signal peptide and generation of an $\mathrm{N}$-terminal propeptide domain and a C-terminal domain. These peptides dimerize through disulfide bonds (latent MSTN) and are then cleaved again by members of the BMP-1/tolloid family of metalloproteinases. This second cleavage results in MSTN propeptide and mature MSTN. The mature MSTN then binds to its cell surface receptors, activin receptor type II or IIb, and activates intracellular members of the SMAD family of signaling proteins, which then translocate to the nucleus and regulate transcription of specific genes in association with diverse transcription factors $(3,4)$.

In addition to the roles of MSTN in skeletal muscle growth, both in vivo and in vitro studies have suggested that MSTN also regulates metabolism by effects on muscle as well as on other metabolic sites such as adipose tissue (5-10). Introduction of MSTN null mutation into different obese strains or deletion of MSTN in mice results in skeletal muscle hypertrophy and, in contrast, reduction in fat accumulation and prevention of insulin resistance $(2-5,7,8,10-13)$. In addition, MSTN gene expression in muscle was upregulated and attenuated by insulin in streptozotocin-induced type 1 diabetic mice (14).

There is also evidence that MSTN is regulated by physical exercise (15-18) which is linked to improved glucose homeostasis and enhanced insulin sensitivity.

In the present study, we evaluated the protein expression of MSTN in two animal models of metabolic diseases, diet-induced obesity and strepzotocin-induced diabetes, in sedentary and exercised rats. 


\section{MATERIAL AND METHODS}

\section{Experimental models}

All procedures were approved by the Ethics Committee of the Federal University of São Carlos (UFSCar, $035 / 2007$ ). Thirty days old male Wistar rats, were initially acclimated in individual polypropylene cages (30 $\mathrm{cm} \times 20 \mathrm{~cm} \times 13 \mathrm{~cm}$ ) under controlled temperature, humidity and lighting (12-h dark/light cycle), with free access to water and standard rodent chow (PRIMOR - São Paulo, Brazil) for 7 days.

\section{Streptozotocin-induced diabetes}

After 7 days of acclimation, animals $(156.7 \pm 3.7 \mathrm{~g})$ were randomly assigned to 2 groups, control rats (C) $(\mathrm{n}=12)$ and diabetic rats $(\mathrm{D})(\mathrm{n}=12)$. Diabetic rats received streptozotocin (STZ, Sigma-Aldrich, St. Louis, USA, $60 \mathrm{mg} / \mathrm{kg}$, intraperitoneally) and control rats received vehicle (sodium citrate, intraperitoneally). Seven days after diabetes induction, control (C) and diabetic (D) animals were randomly assigned to 2 groups: sedentary control (SC) $(\mathrm{n}=5)$ or exercised control (EC) $(\mathrm{n}=6)$ and sedentary diabetic $(\mathrm{SD})(\mathrm{n}=6)$ or exercised diabetic $(\mathrm{ED})(\mathrm{n}=6)$. Glycemia was determined in tail blood using a portable glucometer (Accu-Check glucose meter (Roche Diagnostic, Indianapolis, USA). Soon after diabetes induction, all diabetic animals received 1 U of insulin Lantus ${ }^{\circledR}$ (Sanofi Aventis, Deutschland) intraperitoneally on alternate days to avoid very high glycemia.

\section{Diet-induced obesity}

After 7 days of acclimation, animals $(190 \pm 18.4 \mathrm{~g})$ were randomly assigned to 2 groups, control rats (CG) $(\mathrm{n}=12)$ fed standard rodent chow and rats fed a highfat diet $(\mathrm{HF})(\mathrm{n}=12)$ containing $60 \%$ of kcal as fat (Research Diets, New Brunswick, NJ, USA). Animals and food were weighed daily. After 12 weeks, animals of both groups were randomly divided into 2 groups: sedentary control (SC) $(\mathrm{n}=5)$ or exercised control $(\mathrm{EC})(\mathrm{n}=6)$ and sedentary high-fat $\operatorname{diet}(\mathrm{SHF})(\mathrm{n}=5)$ or exercised HF (EHF) $(n=6)$.

The glucose tolerance test (GTT) was performed before and after exercise training. After an overnight fast, unanesthetized rats were injected intraperitoneally with 1.5 of a $50 \%$ glucose solution per $\mathrm{kg}$ body weight $(\mathrm{BW})$. Blood samples were obtained from the tail vein before injection and at 30,60,90, and 120 min after glucose injection. The insulin tolerance test
(ITT) was also performed before exercise training. After an overnight fast, unanesthetized rats were injected intraperitoneally with human insulin, $\mathrm{l} \mathrm{U} / \mathrm{kg}$ BW. Blood samples were obtained from the tail vein before injection and at $15,30,60$, and $90 \mathrm{~min}$ after the insulin challenge. Blood glucose concentrations were measured with an Accu-Check glucose meter (Roche Diagnostic, Indianapolis, USA).

\section{Exercise protocol}

In both models, rats in the exercise groups were exercised by swimming for $1.5 \mathrm{~h} /$ day, $45 \mathrm{~min}$ at 9:00 AM and $45 \mathrm{~min}$ at 5:00 PM, for 4 weeks. The swimming exercise was performed in plastic barrels $(30 \mathrm{~cm}$ in diameter) filled with water (50 cm deep) maintained at $32-36^{\circ} \mathrm{C}$ and with a weight corresponding to $5 \% \mathrm{BW}$ attached to the tail of each rat. The protocol started with the animals swimming $30 \mathrm{~min}$ on the first day, with a gradual increase so that on the fourth day they could swim for 90 minutes. All animals were towel dried before being returned to their cages. The sedentary groups were placed in shallow water for $5 \mathrm{~min}$ utes, towel dried and then returned to their cages at the same time as the exercised groups.

After the training period, rats were sacrificed by decapitation. White gastrocnemius muscle was dissected, weighed, immediately frozen in liquid nitrogen, and stored at $-80^{\circ} \mathrm{C}$ until analysis.

\section{Western blotting analysis}

MSTN protein levels in gastrocnemius muscle were analyzed by Western Blot according to a method previously described (15). Muscle $(50 \mathrm{mg}$ ) protein was extracted using Tris-Triton buffer $(10 \mathrm{mM}$ Tris, $\mathrm{pH}$ 7.4, $100 \mathrm{mM} \mathrm{NaCl}, 1 \mathrm{mM}$ EDTA, I mM EGTA, $1 \%$ Triton X-100, 10\% glycerol, $0.1 \%$ SDS, $0.5 \%$ deoxycholate) and the protein content of the supernatant was quantified by the BCA method (BCA ${ }^{\mathrm{TM}}$ Protein Assay Kit, Thermo Scientific, USA). Samples ( $250 \mu \mathrm{g}$ of total protein) were separated on $12 \%$ polyacrylamide gel and then transferred to a nitrocellulose membrane. Coomassie Blue staining of the gels confirmed optimal protein transfer. Nitrocellulose membranes were incubated overnight at $4^{\circ} \mathrm{C}$ with antimyostatin primary antibodies (GDF-8 N-19-R sc-6885-R, Santa Cruz Biotechnology, Santa Cruz, CA, USA) diluted 1:200 with Tris buffered saline with Tween added (TBST). Membranes were then washed with TBST and incubated with a 
peroxidase-conjugated secondary antibody (goat antirabbit IgG-HRP: sc-2004). Protein levels were normalized to those of actin (I-19 - sc 1616, Santa Cruz Biotechnology, Santa Cruz, CA, USA). Enhanced chemiluminescence (ECL) Plus Western Blotting Detection Reagents (GE Healthcare Chalfont St. Giles, UK) was used to detect bound antibody.

\section{Data analysis}

Data were analyzed statistically by analysis of variance (ANOVA). Tukey's multiple comparisons test was used for post hoc analysis of between-group comparisons. Data are reported as means \pm SEM. P values $<0.05$ were considered to be statistically significant.

\section{RESULTS}

\section{STZ-induced diabetes}

By the third week of the experiment, the BW of SD animals was significantly lower than that of SC animals $(\mathrm{P}<0.05)$ and this difference was maintained till the end of the experiment. There was no difference in BW between sedentary and exercised animals (data not shown). Before training, diabetic rats had significantly higher glycemia than control rats $(\mathrm{P}<0.05)$. After training, ED animals had significantly lower glycemia than SD animals $(\mathrm{P}<0.05)$. No difference was observed between sedentary and exercised control animals after training (Figure 1).

Diabetes induced a significant decrease of gastrocnemius muscle weight in sedentary diabetic animals compared to sedentary control animals $(\mathrm{P}<0.05)$. However, exercise induced an increase in gastrocne-

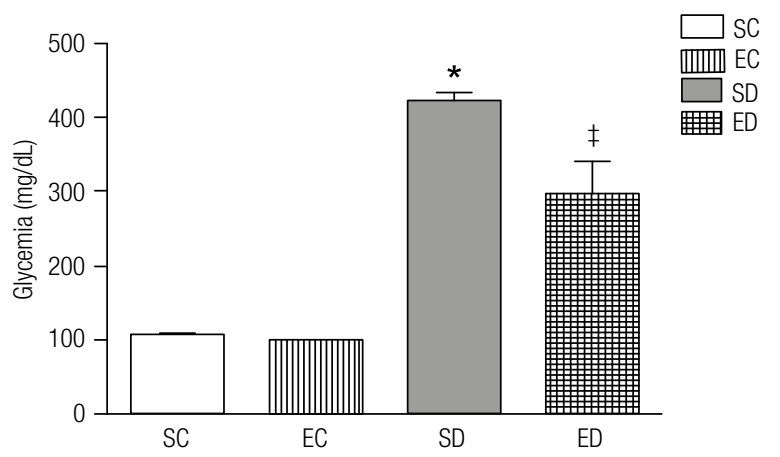

Figure 1. Glycemia of STZ-induced diabetic animals.

Glycemia $(\mathrm{mg} / \mathrm{dL})$ at the end of the training period in the sedentary control group (SC), exercised control group (EC), sedentary diabetic group (SD) and exercised diabetic group (ED). Values are means \pm SEM for $n=5-6$ per group. $\mathrm{P}<0.05$, *SC vs. SD; ${ }^{\mathrm{S} D}$ vs. ED. mius muscle weight in diabetic animals compared to sedentary diabetic animals $(\mathrm{P}<0.05)$ (Figure 2$)$.

The precursor and processed forms of MSTN in gastrocnemius muscle were significantly higher in sedentary diabetic rats than in sedentary control rats $(\mathrm{P}<$ $0.05)$. However, the precursor form was significantly lower in diabetic exercised animals compared to diabetic sedentary animals $(\mathrm{P}<0.05)$ and the processed form did not change in these two groups (Figures $3 \mathrm{~A}$ and $3 \mathrm{~B})$.

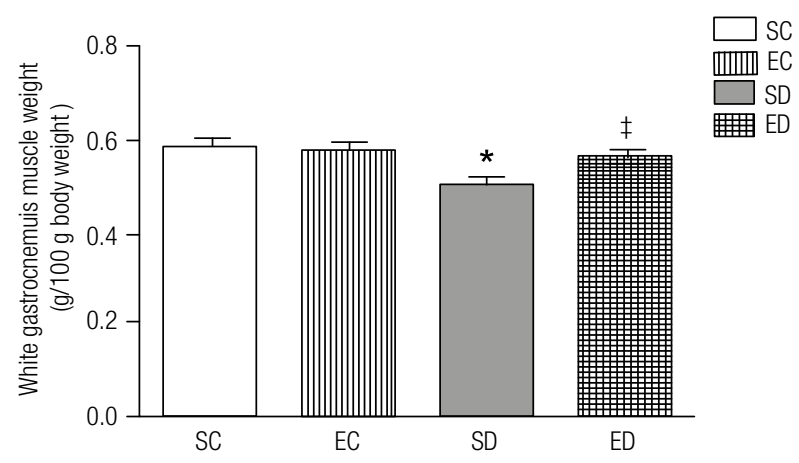

Figure 2. Muscle weight of STZ-induced diabetic animals: weight of white gastrocnemius muscle ( $\mathrm{g} / 100 \mathrm{~g}$ body weight) of the sedentary control group (SC), exercised control group (EC), sedentary diabetic group (SD) and exercised diabetic group (ED). Values are means \pm SEM for $n=5-6$ per group. $\mathrm{P}<0.05,{ }^{*} \mathrm{SC}$ vs. SD; ${ }^{\mathrm{S} S D}$ vs. ED.

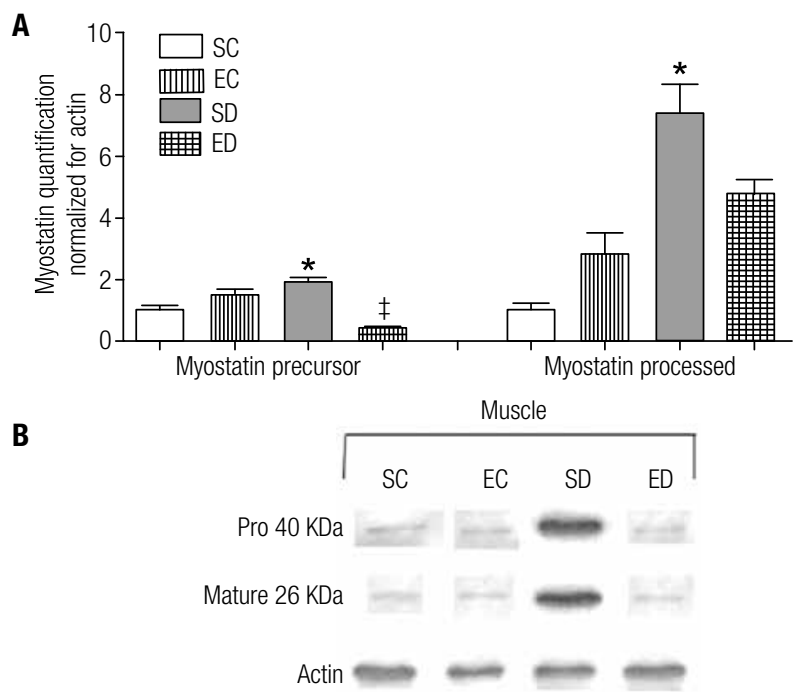

Figure 3 (A) MSTN protein expression in STZ-induced diabetic animals: expression of MSTN protein precursor (A) and processed (B) forms in white gastrocnemius muscle of the sedentary control group (SC), exercised control group (EC), sedentary diabetic group (SD) and exercised diabetic group (ED). Values are means \pm SEM for $n=5-6$ rats per group. * $P<0.05$, sedentary diabetic group (SD) VS. sedentary control group (SC); ${ }^{\ddagger} \mathrm{P}<0.05$, exercised diabetic group (ED) vs. sedentary diabetic group (SD). (B). Representative Western blotting of MSTN protein forms in white gastrocnemius muscle of sedentary control group (SC), exercised control group (EC), sedentary diabetic group (SD) and exercised diabetic group (ED). 


\section{Diet-induced obesity}

A high-fat diet induced a significant increase in $\mathrm{BW}$ in the rats fed a high-fat diet compared to controls $(\mathrm{P}<$ 0.05). After training, both exercised high-fat diet-fed and control animals had significantly lower body weight compared to sedentary high-fat diet-fed and control animals, respectively $(\mathrm{P}<0.05)$ (Figure 4$)$. There was no difference in gastrocnemius muscle weight between high-fat diet-fed and control animals, sedentary or exercised. High-fat diet induced significantly increase in mesenteric, retroperitoneal and epididymal adipose tissue mass in sedentary high-fat fed diet animals compared to sedentary control animals.

Before training, rats fed a high-fat diet showed a significantly higher glucose response to both insulin and glucose injections than control rats (Figure 5). After exercise, the glucose response to glucose injection was

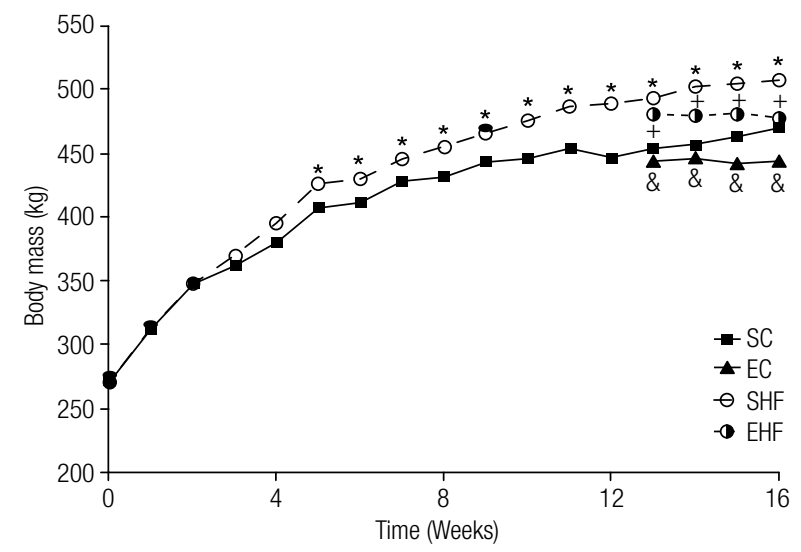

Figure 4. Body mass in animals fed a high-fat diet: body mass of the sedentary control group (SC), sedentary high-fat diet group (SHF), exercised control group (EC) and exercised high-fat diet group (EHF). Values are means \pm SEM for 5-6 rats per group. $p<0.05$, ${ }^{*}$ SC vs SHF; + SHF vs. EHF; \& SHF vs. EHF.

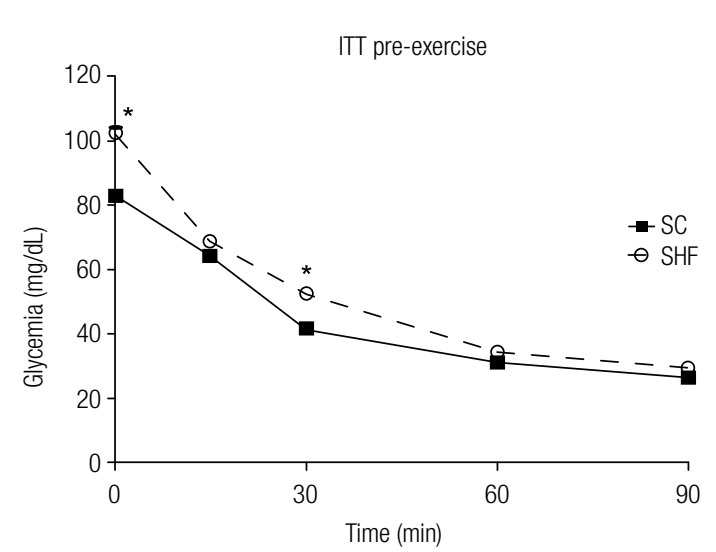

Figure 5. Insulin test tolerance in animals fed a high-fat diet (ITT) preexercise: sedentary control group (SC), sedentary high-fat diet group (SHF). Values are means \pm SEM for $5-6$ rats per group. $p<0.05,{ }^{*}$ SC vs. SHF. significantly lower than the previous test response in rats fed a high-fat diet $(\mathrm{P}<0.05)$ (Figure 6). No difference was observed between the pre- and post-exercise glucose response to glucose injection in control rats.

No difference in the expression of MSTN protein precursor and processed forms was observed between the various groups (Figures 7A and 7B).

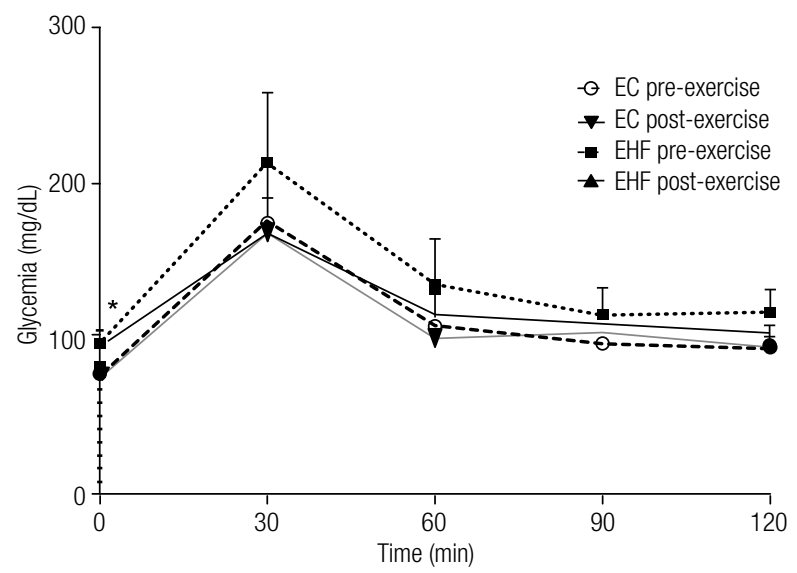

Figure 6. Glucose tolerance test in animals fed a high-fat diet: glycemia profile in the glucose tolerance test (GTT) pre- and post-exercise training. Values are means \pm SEM for $n=5-6$ rats per group. ${ }^{*} P<0.05$, exercised control group (EC) pre-exercise vs. exercised high-fat diet group (EHF) pre-exercise; \& $P<0.05$, exercised high-fat diet group (EHF) pre-exercise vs. exercised high-fat diet group (EHF) post-exercise.

A

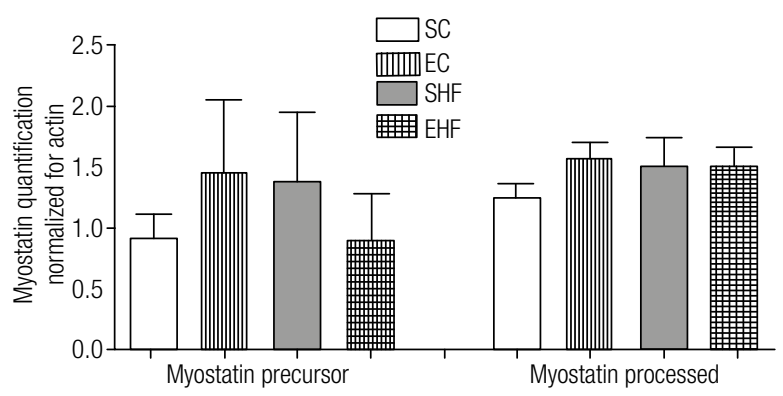

B

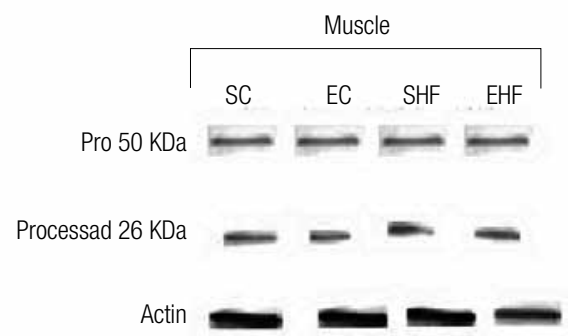

Figure 7 (A) MSTN protein expression in animals fed a high-fat diet: Expression of MSTN protein precursor (A) and processed (B) forms in white gastrocnemius muscle of the sedentary control group (SC), sedentary high-fat diet group (SHF), exercised control group (EC) and exercised high-fat diet group (EHF). Values are means \pm SEM for $n=5-6$ rats per group. (B). RepresentativeWestern blotting of MSTN protein forms in white gastrocnemius muscle in the sedentary control group (SC), sedentary high-fat diet group (SHF), exercised control group (EC) and exercised high-fat diet group (EHF). 


\section{DISCUSSION}

The present results show that the expression of MSTN protein varies in muscle of both sedentary and exercised rats with streptozotocin-induced diabetes, but not in animals with high fat diet-induced obesity.

Training improved postprandial glycemia in diabetic animals and glucose tolerance in rats fed a high-fat diet. It has been demonstrated that exercise improves insulin sensitivity in diet-induced obese rats by improving insulin and AMP-activated protein kinase (AMPK) signaling pathways $(19,20)$. In addition, there is evidence suggesting that exercise increases insulin secretion and has a protective effect in STZ-induced diabetes by decreasing oxidative stress and preserving pancreatic beta cell integrity (21-23) observed was increasing muscle mass by myostatin inhibition elevates basal metabolic rate dystrophic muscle by myostatin blockade. Thus clinical applications; for example, treatment of type II diabetics by myostatin inhibition to altering muscle mass would hypothetically result in increased whole body energy consumption, reduce peripheral fat mass and potentially lower blood glucose (24).

In STZ-induced diabetic rats, the expression of both MSTN protein precursor and mature forms was increased in skeletal muscle. The expression of MSTN protein precursor was decreased in exercised diabetic rats, but the expression of mature protein did not change. It has been previously demonstrated that MSTN mRNA expression $(14,25)$ in gastrocnemius muscle is increased in STZ-induced diabetes, suggesting that upregulation of MSTN expression contributes to muscle atrophy in insulin deficiency. However, Barazzoni and cols., (26) have reported that MSTN mRNA expression is not altered in the lateral gastrocnemius and soleus muscles of STZ-induced diabetic rats with or without insulin replacement. The discrepancy between these studies may be due to different experimental designs.

The different profile of expression of MSTN precursor and mature forms in muscle of rats with streptozotocin-induced diabetes could possibly be explained by the modulation of proteolysis processing of MSTN. In fact, it has been demonstrated that BMP-1/metalloproteases are involved in the regulation of MSTN maturation (27-29). Recent data have shown that the increased insulin sensitivity and adipose tissue reduction observed in MSTN null mice is an indirect result of metabolic changes in skeletal muscle (6). Previous studies have shown that MSTN may signal through Akt phosphorylation and thus affect insulin sensitivity in muscle. Previous reports suggest that myostatin protein concentrations are regulated not only at the transcriptional level but by posttranscriptional mechanisms as well (27-29). In muscle and adipose tissue from MSTN null mice and transgenic mice overexpressing MSTN propeptide, insulin signaling was increased by a higher level of Akt phosphorylation $(6,30,31)$. One mechanism by which myostatin stimulation induces hypoglycaemia is by increasing mRNA activity of several glucose-regulating proteins, including GLUTl and GLUT4, IL6, hexokinase and phosphorylated adenosine triphosphate kinase - AMPK, thereby increasing cellular glucose uptake (32).

In the present study, MSTN protein expression did not vary in muscle of obese rats. However, an increase of MSTN has been shown in muscle of extremely obese women (33). Although the expression of MSTN protein did not vary in the gastrocnemius muscle of control and obese animals, sedentary or exercised groups, the presence of both precursor and mature forms of MSTN in muscle suggests the occurrence of proteolysis processing of MSTN in this tissue.

We have previously shown the profile of MSTN mRNA expression in obese and exercised animals (3) which differs from the MSTN protein expression profile shown in the present study. The discrepancy observed in MSTN mRNA and protein expression has been observed in other studies and suggests that MSTN protein concentrations are regulated not only at the transcriptional level but by post-transcriptional mechanisms as well $(4,34)$.

In conclusion, the results demonstrate that exercise can modulate the increased muscle expression of MSTN protein in diabetic rats and suggest that MSTN may participate in energy homeostasis. However, future studies are needed to elucidate the role and mechanisms of action of myostatin.

Acknowledgements: this study was supported by Fundação de Amparo à Pesquisa do Estado de São Paulo (Fapesp) and Conselho Nacional de Desenvolvimento Científico e Tecnológico (CNPq).

Disclosure: no potential conflict of interest relevant to this article was reported.

\section{REFERENCES}

1. McPherron AC, Lee SJ. Double muscling in cattle due to mutations in the myostatin gene. Proc Natl Acad Sci U S A. 1997;94(23):12457-61. 
2. Allen DL, Cleary AS, Speaker KJ, Lindsay SF, Uyenishi J, Reed JM, et al. Myostatin, activin receptor llb, follistatin-like-3 gene expression are altered in adipose tissue and skeletal muscle of obese mice. Am J Physiol Endocrinol Metab. 2008;294(5):E918-27.

3. Lee SJ. Regulation of muscle mass by myostatin. Annu Rev Cell Dev Biol. 2004;20:61-86.

4. McFarlane C, Langley B, Thomas M, Hennebry A, Plummer E, Nicholas $\mathrm{G}$, et al. Proteolytic processing of myostatin is auto-regulated during myogenesis. Dev Biol. 2005;283(1):58-69.

5. Feldman BJ, Streeper RS, Farese RV Jr, Yamamoto KR. Myostatin modulates adipogenesis to generate adipocytes with favorable metabolic effects. Proc Natl Acad Sci U S A. 2006;103(42):15675-80.

6. Guo T, Jou W, Chanturiya T, Portas J, Gavrilova O, McPherron AC. Myostatin inhibition in muscle, but not adipose tissue, decreases fat mass and improves insulin sensitivity. PLoS One. 2009;4(3):e4937.

7. Kim HS, Liang L, Dean RG, Hausman DB, Hartzell DL, Baile CA. Inhibition of preadipocyte differentiation by myostatin treatment in 3T3-L1 cultures. Biochem Biophys Res Commun. 2001 Mar 9;281(4):902-6.

8. Rebbapragada A, Benchabane H, Wrana JL, Celeste AJ, Attisano L. Myostatin signals through a transforming growth factor beta-like signaling pathway to block adipogenesis. Mol Cell Biol. 2003;23(20):7230-42.

9. Zhao B, Wall RJ, Yang J. Transgenic expression of myostatin propeptide prevents diet-induced obesity and insulin resistance. Biochem Biophys Res Commun. 2005;337(1):248-55.

10. Zimmers TA, Davies MV, Koniaris LG, Haynes P, Esquela AF, Tomkinson $\mathrm{KN}$, et al. Induction of cachexia in mice by systemically administered myostatin. Science. 2002;296(5572):1486-8.

11. Bueno PG, Bassi D, Contrera DG, Carnielli HM, Silva RN, Nonaka KO, et al. Post-exercise changes in myostatin and actRIIB expression in obese insulin-resistant rats. Mol Cell Endocrinol. 2011;339(1-2):159-64.

12. Lin J, Arnold HB, Della-Fera MA, Azain MJ, Hartzell DL, Baile CA. Myostatin knockout in mice increases myogenesis and decreases adipogenesis. Biochem Biophys Res Commun. 2002;291(3):701-6.

13. McPherron AC, Lee SJ. Suppression of body fat accumulation in myostatin-deficient mice. J Clin Invest. 2002;109(5): 595-601.

14. ChenY, Cao L, Ye J, Zhu D. Upregulation of myostatin gene expression in streptozotocin-induced type 1 diabetes mice is attenuated by insulin. Biochem Biophys Res Commun. 2009;388(1):112-6.

15. Costelli P, Muscaritoli M, Bonetto A, Penna F, Reffo P, Bossola M, et al. Muscle myostatin signalling is enhanced in experimental cancer cachexia. Eur J Clin Invest. 2008;38(7):531-8.

16. Heinemeier KM, Olesen JL, Schjerling $P$, Haddad $F$, Langberg $H$, Baldwin KM, et al. Short-term strength training and the expression of myostatin and IGF-I isoforms in rat muscle and tendon: differential effects of specific contraction types. J Appl Physiol (1985). 2007;102(2):573-81.

17. Hulmi JJ, Ahtiainen JP, KaasalainenT, Pollanen E, Häkkinen K, Alen $M$, et al. Postexercise myostatin and activin Ilb mRNA levels: effects of strength training. Med Sci Sports Exerc. 2007;39(2):289-97.

18. Matsakas A, Bozzo C, Cacciani N, Caliaro F, Reggiani C, Mascarello $\mathrm{F}$, et al. Effect of swimming on myostatin expression in white and red gastrocnemius muscle and in cardiac muscle of rats. Exp Physiol. 2006 Nov;91(6):983-94.
19. Pauli JR, Ropelle ER, Cintra DE, Carvalho-Filho MA, Moraes JC, De Souza CT, et al. Acute physical exercise reverses S-nitrosation of the insulin receptor, insulin receptor substrate 1 and protein kinase B/Akt in diet-induced obese Wistar rats. J Physiol. 2008;586(2):659-71.

20. Yaspelkis BB, Lessard SJ, Reeder DW, Limon JJ, Saito M, Rivas DA, et al. Exercise reverses high-fat diet-induced impairments on compartmentalization and activation of components of the insulin-signaling cascade in skeletal muscle. Am J Physiol Endocrinol Metab. 2007;293(4):E941-9.

21. Coskun O, Ocakci A, Bayraktaroglu T, Kanter M. Exercise training prevents and protects streptozotocin-induced oxidative stress and beta-cell damage in rat pancreas. Tohoku J Exp Med. 2004 Jul;203(3):145-54.

22. Huang HH, Farmer K, Windscheffel J, Yost K, Power M, Wright $\mathrm{DE}$, et al. Exercise increases insulin content and basal secretion in pancreatic islets in type 1 diabetic mice. Exp Diabetes Res. 2011;2011:481427.

23. Bogdanovich S, Krag TO, Barton ER, Morris LD, Whittemore LA, Ahima RS, et al. Functional improvement of dystrophic muscle by myostatin blockade. Nature. 2002;420(6914):418-21.

24. Elliott B, Renshaw D, Getting S, Mackenzie R. The central role of myostatin in skeletal muscle and whole body homeostasis. Acta Physiol (Oxf). 2012;205(3):324-40.

25. Dutra DB, Bueno PG, Silva RN, Nakahara NH, Selistre-Araujo HS, Nonaka KO, et al. Expression of myostatin, myostatin receptors and follistatin in diabetic rats submitted to exercise. Clin Exp Pharmacol Physiol. 2012;39(5):417-22.

26. Barazzoni R, Zanetti M, Bosutti A, Stebel M, Cattin L, Biolo G, et al. Myostatin expression is not altered by insulin deficiency and replacement in streptozotocin-diabetic rat skeletal muscles. Clin Nutr. 2004;23(6):1413-7.

27. Anderson $\mathrm{SB}$, Goldberg AL, Whitman M. Identification of a novel pool of extracellular pro-myostatin in skeletal muscle. J Biol Chem. 2008;283(11):7027-35.

28. Huet C, Li ZF, Liu HZ, Black RA, Galliano MF, Engvall E. Skeletal muscle cell hypertrophy induced by inhibitors of metalloproteases; myostatin as a potential mediator. Am J Physiol Cell Physiol. 2001;281(5):C1624-34.

29. Lee SJ. Genetic analysis of the role of proteolysis in the activation of latent myostatin. PLoS ONE. 2008;3(2):e1628.

30. Tu P, Bhasin S, Hruz PW, Herbst KL, Castellani LW, Hua N, et al. Genetic disruption of myostatin reduces the development of proatherogenic dyslipidemia and atherogenic lesions in Ldlr null mice. Diabetes. 2009;58(8):1739-48.

31. Chen Y,Ye J, Cao L, ZhangY, XiaW, Zhu D. Myostatin regulates glucose metabolism via the AMP-activated protein kinase pathway in skeletal muscle cells. Int J Biochem Cell Biol. 2010;42(12):2072-81.

32. Hittel DS, Berggren JR, Shearer J, Boyle K, Houmard JA. Increased secretion and expression of myostatin in skeletal muscle from extremely obese women. Diabetes. 2009;58(1):30-8.

33. Smith IJ, Aversa Z, Alamdari N, Petkova V, Hasselgren PO. Sepsis downregulates myostatin mRNA levels without altering myostatin protein levels in skeletal muscle. J Cell Biochem. 2010;111(4):1059-73. 